\title{
Correspondence
}

http://dx.doi.org/10.11646/phytotaxa.172.1.8

\section{Thismia gigantea, a new combination in Thismia (Thismiaceae)}

\author{
MICHAL HRONEŠ \\ Department of Botany, Faculty of Science, Palacký University, Šlechtitelů 11, CZ-78371 Olomouc, Czech Republic \\ Email:michal.hrones@gmail.com
}

Thismia Griffith (1844: 221; Thismiaceae J. Agardh, 1858: 99) comprises about 55 species (e.g. Tsukaya \& Okada 2012; Dančák et al. 2013; Lưu et al. 2014). The greatest species diversity is situated in southeastern Asia and in the northern parts of South America (Merckx et al. 2013). Both morphological and molecular data suggest that the genus is not monophyletic being represented by at least two evolutionary lineages, the neotropical Thismia subg. Ophiomeris (Miers) Maas \& H.Maas (1986: 144) and the rest of the species in a broadly defined subg. Thismia (Merckx et al. 2006; Merckx \& Smets 2014).

At the end of the $19^{\text {th }}$ century, there were tendencies to split Thismia into several genera, such as Geomitra Beccari (1878: 250), Sarcosiphon Blume (1850: 65) and Scaphiophora Schlechter (1921: 39). As currently circumscribed (Maas 1998; Merckx et al. 2013), Thismia contains most of these previously described Asian genera. Scaphiophora was established for Thismia appendiculata Schlechter (1918: 202) by the German botanist Rudolf Schlechter on the basis of the flowers crowned by one, erect, thick column bearing at its apex three connate lobes (Schlechter 1921; Jonker 1948). A second species, Scaphiophora gigantea Jonker (1938: 257), was described from the Philippines twenty years later. As noted above, the genus Scaphiophora is now considered to be synonymous with Thismia. Consequently the name Thismia gigantea was used recently by Merckx et al. (2013) and Merckx \& Smets (2014), without formal validation of the name. Therefore, a new combination is proposed:

Thismia gigantea (Jonker) Hroneš, comb. nov.

Basionym:- Scaphiophora gigantea Jonker (1938: 257)

Type:-PHILIPPINES. Luzon, Province of Laguna, Makiling, A. Loher s.n. (holotype M).

\section{Acknowledgements}

Creation of this manuscript was supported by internal fund from Palacký University IGA PrF 2014001. Martin Dančák is acknowledged for helpful comments on the manuscript.

\section{References}

Agardh, J.G. (1858) Theoria systematis plantarum. C.W.K. Gleerup, Lund.

Beccari, O. (1878) Descrizione di una nuova e singolare pianta parassita. Malesia 1: 238-240.

Blume, C.L. (1850) Stirpium exoticarum novarum vel minus cognitarum ex vivis aut siccis brevis expositio et descriptio, additis figuris. Museum Botanicum Lugduno-Batavum 1(5): 65-80. http://dx.doi.org/10.5962/bhl.title.274

Dančák, M, Hroneš, M., Sochor, M., Kobrlová, L., Hédl, R., Hrázský, Z., Vildomcová, A., Sukri, R.S. \& Metali, F. (2013) A new species of Thismia (Thismiaceae) from Brunei Darussalam, Borneo. Phytotaxa 125: 33-39.

http://dx.doi.org/10.11646/phytotaxa.125.1.5

Griffith, W. (1844) On the root parasites referred by authors to Rhizantheae and their allies. Proceedings of the Linnean Society of London 1: $216-221$.

Jonker, F.P. (1938) A monograph of the Burmanniaceae. Mededeelingen van het Botanisch Museum en Herbarium van de Rijks Universiteit te Utrecht 51: 1-279. 
Jonker, F.P. (1948) Burmanniaceae. In: van Steenis, C.G.G.J. (ed.), Flora Malesiana I, 4. Noordhoff-Kolf, Leiden, pp. $13-26$.

Lưu, H.T., Tịch, N.T., Trần, G., Đinh, Q.D., Vũ, N.L., Bách, X.N., Dung, T.T.N. \& Trung, T.N. (2014) Thismia okhaensis (Thismiaceae) - a new fairy lantern from Vietnam. Phytotaxa 164: 190-194.

http://dx.doi.org/10.11646/phytotaxa.164.3.4

Maas, P.J.M., Maas-van de Kamer, H., van Benthem, J., Snelders, H.C.M. \& Rübsamen, T. (1986) Burmanniaceae. In: Organization for Flora Neotropica (eds), Flora Neotropica 42. New York Botanical Garden, New York, pp 1-189.

Maas-van de Kamer, H. (1998) Burmanniaceae. In: Kubitzki, K. \& Huber, H. (eds.), Families and genera of vascular plants. Monocotyledons: Lilianae (except Orchidaceae). Springer, Berlin, pp. 154-164.

Merckx, V.S.F.T., Schols, P., Maas-van de Kamer, H.M., Maas, P., Huysmans, S. \& Smets, E. (2006) Phylogeny and evolution of Burmanniaceae (Dioscoreales) based on nuclear and mitochondrial data. American Journal of Botany 93: 1684-1698.

http://dx.doi.org/10.3732/ajb.93.11.1684

Merckx, V.S.F.T., Freudenstein, J.V., Kissling, J., Christenhusz, M.J.M., Stotler, R.E., Crandall-Stotler, B., Wickett, N., Rudall, P.J., Maasvan de Kamer, H. \& Maas, P.J.M. (2013) Taxonomy and classification. In: Merckx, V.S.F.T. (ed.), Mycoheterotrophy: the biology of plants living on fungi. Springer, New York, pp. 19-101.

http://dx.doi.org/10.1007/978-1-4614-5209-6_2

Merckx, V.S.F.T. \& Smets, E. (2014): Thismia americana, the 101st anniversary of a botanical mystery. International Journal of Plant Sciences 175: 165-175.

http://dx.doi.org/10.1086/674315

Schlechter, F.R.R (1918) Eine neue papuasische Burmanniacee. Botanische Jahrbücher für Systematik, Pflanzengeschichte und Pflanzengeographie 55: 202-203.

Schlechter, F.R.R (1921) Die Thismieae. Notizblatt des Königlichen botanischen Gartens und Museums zu Berlin 8: 31-45.

Tsukaya, H. \& Okada, H. (2012) A new species of Thismia (Thismiaceae) from West Kalimantan, Borneo. Systematic Botany $37: 53-57$. http://dx.doi.org/10.1600/036364412x616639 